\title{
O homem na busca dos serviços de atenção primária em saúde na cidade de
}

\section{Imperatriz-MA}

\author{
The man in search of primary health care services in the city of Imperatriz-MA \\ El hombre em busca de servicios de atención primaria de salud en la ciudad de Imperatriz-MA
}

Recebido: 19/12/2021 | Revisado: 27/12/2021 | Aceito: 13/01/2022 | Publicado: 15/01/2022

Jeovanna Ferreira Miranda

ORCID: https://orcid.org/0000-0003-4366-8396 Universidade Ceuma, Brasil E-mail: jeovanna_miranda@ hotmail.com

Marcelo Pereira de Araújo

ORCID: https://orcid.org/0000-0003-3673-5685 Universidade Ceuma, Brasil

E-mail: marceloparaujo06@ hotmail.com

Karyne Gleyce Zemf Oliveira

ORCID: https://orcid.org/0000-0002-3676-7719 Universidade Ceuma, Brasil

E-mail: karyne_zemf@hotmail.com

\begin{abstract}
Resumo
Objetivo: Compreender os fatores relacionados a não procura dos homens com idade entre 20 e 59 anos aos serviços básicos de saúde na cidade de Imperatriz - MA. Metodologia: estudo quantitativo exploratório que foi desenvolvido através de um questionário semiestruturado com perguntas objetivas aplicado por meio da plataforma Forms. Resultados: 169 homens responderam o instrumento, sendo que destes, 86,39\% estavam com a idade entre 20 e 40 anos, maioria eram solteiros $69,23 \%$. Sobre a busca do serviço de atenção primária para prevenção de doenças ou promoção da saúde, 39,06\%, apontaram que somente buscam quando sente algum sintoma, e 42,60\% relatam que quando observam algum sinal ou sintoma, procuram como porta de entrada no sistema de saúde à atenção terciaria para resolução do problema. Conclusão: Diante dos resultados obtidos percebe-se que os homens possuem hesitação para buscar os serviços de saúde. Nesse contexto, há necessidade da execução efetivas das políticas públicas para esse público afim de que se tenha uma procura pela atenção básica como forma preventiva.
\end{abstract}

Palavras-chave: Atenção primária; Homem; Prevenção.

\begin{abstract}
Objective: To understand the factors related to men aged between 20 and 59 years not seeking basic health services in the city of Imperatriz - MA. Methodology: exploratory quantitative study that was developed through a semistructured questionnaire with objective questions applied through the Forms platform. Results: 169 men answered the instrument, and of these, $86.39 \%$ were aged between 20 and 40 years, most were single $69.23 \%$. Regarding the search for the primary care service for disease prevention or health promotion, $39.06 \%$ indicated that they only seek it when they feel a symptom, and $42.60 \%$ report that when they observe any sign or symptom, they look for it as a gateway in the health system to tertiary care to solve the problem. Conclusion: Based on the results obtained, it is clear that men are hesitant to seek health services. In this context, there is a need for the effective execution of public policies for this public in order to have a demand for primary care as a preventive form.
\end{abstract}

Keywords: Primary care; Man; Prevention.

\section{Resumen}

Objetivo: Comprender los factores relacionados con hombres de 20 a 59 años que no buscan servicios básicos de salud en la ciudad de Imperatriz - MA. Metodología: estudio cuantitativo exploratorio que se desarrolló a través de un cuestionario semiestructurado con preguntas objetivas aplicadas a través de la plataforma Forms. Resultados: 169 hombres respondieron al instrumento, de estos, el 86,39\% tenían entre 20 y 40 años, la mayoría eran solteros 69,23\%. En cuanto a la búsqueda del servicio de atención primaria para la prevención de enfermedades o promoción de la salud, el 39,06\% indicó que solo lo busca cuando siente un síntoma, y el 42,60\% informa que cuando observa algún signo o síntoma lo busca como puerta de entrada en del sistema de salud a la atención terciaria para solucionar el problema. Conclusión: A partir de los resultados obtenidos, es evidente que los hombres dudan en acudir a los servicios de salud. En este contexto, existe la necesidad de la ejecución efectiva de políticas públicas para este público a fin de tener una demanda de atención primaria como forma preventiva.

Palabras clave: Atención primaria; Hombre; Prevención. 


\section{Introdução}

No Brasil, os homens vivem em média 7,1 anos menos do que as mulheres (Brasil, 2016), sendo isto atribuída para as principais causas de mortes para esse público com a faixa etária entre 20 e 59 anos a causas externas (acidentes, homicídios e quedas) com 35,2 \%, doenças do aparelho circulatório com 17,9\%, neoplasias com 11,8\% e doenças do aparelho digestório representado por 7,6\% (Brasil, 2012).

No Maranhão predomina como principais causas de mortes nos homens com idade de 20 a 59 anos: 41,3\% devido causas externas, $19 \%$ doenças do aparelho circulatório, 7,7\% neoplasias e 7,3\% de doenças do aparelho digestório (Brasil, 2012).

No cotidiano da APS, é evidente a presença de mais mulheres do que homens, sendo que estes só procuram atendimento quando são acometidos por doenças, enquanto o público feminino busca atividades preventivas. A escassa procura dos homens e consequente sobrecarga pelos serviços da atenção especializada provoca custos que muitas vezes poderia ser evitado (Silva, 2018).

Diversas ações estão sendo realizadas para prevenção da morbimortalidade dos homens, como as adotadas pela Atenção Primária à Saúde (APS) através da Política Nacional de Atenção Integral à Saúde do Homem (PNAISH) lançada em 2009 pelo Ministério da Saúde (Brasil, 2012). Contudo, nota-se a baixa procura da população masculina na APS, o que favorece a vulnerabilidade desses indivíduos para doenças (Fontes et al., 2010).

Os homens não são captados pelos serviços de atenção à saúde, sobretudo aqueles relacionados à assistência primária. O acesso masculino as ações desenvolvidas no sistema de saúde são muitas vezes, a partir da atenção ambulatorial e hospitalar média e alta complexidade, configurando uma inadequação do nível básico de cuidado o que contribui para o aumento da morbimortalidade masculino (Moreira \& Carvalho, 2016).

Segundo Teixeira e Cruz (2016), a não adesão do público masculino se faz pela falta de percepção da sintomatologia para problemas físicos e/ou mentais, assim como a falta de tempo devido ao trabalho. Homens que só procuram auxílio médico em situações extremas, devido se julgarem imunes, não reconhecendo a doença como algo a que estão expostos é outo fator para a falta de procura dos serviços de saúde. Com isso, a qualidade da saúde masculina segue ditada por aspectos culturais, educacionais e sociais (Ferreira et al., 2013). Objetivo deste trabalho foi compreender os fatores relacionados a não procura dos homens de 20 a 59 anos aos serviços básicos de saúde na cidade de Imperatriz - MA.

\section{Metodologia}

A prática da investigação científica realiza-se sob as mais diversas perspectivas epistemológicas e de abordagens, como as da pesquisa quantitativa e da pesquisa qualitativa. A quantitativa apoia em um modelo de conhecimento chamado positivista, em que prevalece a preocupação estatístico-matemática e tem a pretensão de ter acesso racional à essência dos objetos e fenômenos examinados. Tem como característica a configuração experimental (Marconi \& Lakatos, 2017).

Com base na população estudada, a amostra do estudo foi aleatória por conveniência, não probabilística, sendo constituída por homens com idade maior ou igual a 20 anos e menor ou igual a 59 anos que residem na cidade de Imperatriz, estado Maranhão. O instrumento foi um questionário semiestruturado com perguntas objetivas e de sequência lógica. A aplicação ocorreu por meio de aplicativo da plataforma Forms no período de junho a agosto de 2021.

As variáveis abordadas foram realizadas por blocos: condições Socioeconômicas, condições de Saúde, comportamento de saúde.

Para análise dos dados foi utilizado o programa Microsoft Office Exce ${ }^{\circledR} 2016$ e que foram tratados por meio de análise Quantitativa Progressiva (porcentagem). Também foram utilizado o Microsoft Office Word ${ }^{\circledR} 2016$ para elaboração de texto. Os 
dois programas foram posteriormente utilizados para a organização dos dados em forma de gráficos e tabelas para discussão conforme a literatura.

A pesquisa realizada seguiu as normas éticas estabelecidos na Resolução n ${ }^{\circ} 466$ de 12 de dezembro de 2012 que trata sobre pesquisas e testes realizados em seres humanos. A coleta de dados foi realizada após aprovação do projeto de pesquisa da enviada ao Comitê de Ética e Pesquisa da Universidade Ceuma, sendo aprovada sob o número CAAE 4.005.666.

Para responder as perguntas na plataforma, os participantes tiveram que concordar com os tópicos do termo de consentimento livre e esclarecido através do aceite online.

\section{Resultados e Discussão}

A população amostral do presente trabalho foi de 169 homens da cidade de Imperatriz- Maranhão. Sendo que a maioria dos homens $86,39 \%$ estavam com a idade entre 20 e 40 anos, $69,23 \%$ eram solteiros e de religião católica $39,05 \%$ seguido por 33,73 evangélica (Tabela 1).

Tabela 1. Distribuição dos homens de acordo com idade, estado civil e escolaridade, Imperatriz - MA, 2021.

\begin{tabular}{lcc}
\hline Variável & N & Percentual (\%) \\
\hline Idade & 146 & 86,39 \\
20 a 40 anos & 23 & 13,61 \\
41 a 60 anos & 169 & 100 \\
Total & & \\
Estado Civil & 117 & 69,23 \\
Solteiro & 42 & 24,85 \\
Casado & 10 & 5,92 \\
Outro & 169 & 100 \\
Total & & \\
Escolaridade & 53 & 31,36 \\
Ensino médio & 59 & 34,91 \\
Ensino superior incompleto & 57 & 33,73 \\
Ensino superior completo & 169 & 100 \\
Total & & \\
\hline
\end{tabular}

Fonte: Autores.

Na pesquisa de Czorny et al., (2017), relata que o fator idade induz uma população a assumir meios de cuidados preventivos, visto que, devido às doenças crônicas não transmissíveis não possuírem um agente etiológico e à pouca compreensão dos processos fisiológicos, a prevenção para esse tipo de doença torna-se um desafio. Homens na fase produtiva raramente buscam os serviços de saúde por desconhecimento da importância ou ausência de preocupação com as ações de promoção e prevenção da saúde, medo da doença e fatores institucionais relacionados com a organização de horário de atendimento das unidades básicas de saúde (Barbosa, 2018).

O estado civil interfere na saúde do homem, tendo em vista que, segundo Francisco e Maria (2019), na maioria das vezes, os homens somente procuram os serviços de saúde quando estimulados pelo cônjuge, sendo o matrimônio conceituado como um fator de proteção de riscos e agravos de saúde para os homens.

A variável escolaridade é fundamental na efetivação de programas específicos para a saúde do homem, pois é notório que o menor grau de escolaridade complica a aplicação de programas preventivos, sobretudo os da atenção primária, considerados de amplo poder resolutivo para vários problemas de saúde, por serem voltados à promoção da saúde e prevenção de doenças (Albuquerque \& Farias, 2019). 
Quando questionados sobre a renda familiar, demonstrou-se que 24,85\% 1 a 2 salários, 28,40\% possuem 3 a 5 salários como renda familiar, e 31,36\% acima de 6 salários. A situação de trabalho dos entrevistados eram: 45,56\% estudantes e $42,01 \%$ trabalham de maneira formal, tendo $12,43 \%$ de trabalho informal.

Tabela 2. Caracterização da situação de trabalho e renda familiar, Imperatriz - MA, 2021

\begin{tabular}{lcc}
\hline Variável & N & Percentual (\%) \\
\hline Situação de Trabalho & 71 & 42,01 \\
Formal & 77 & 45,56 \\
Estudante & 21 & 12,43 \\
Informal & 169 & 100 \\
Total & & \\
Renda familiar & 42 & 24,85 \\
1 a 2 salários & 48 & 28,40 \\
3 a 5 salários & 53 & 31,36 \\
Acima de 6 salários & 25 & 14,79 \\
Não declarar & 168 & 100 \\
Total & & \\
\hline
\end{tabular}

Fonte: Autores.

A condição econômica e nível de escolaridade consistem como preditores do uso de serviços de saúde, por se relacionarem ao nível de conhecimento sobre saúde e à adoção de comportamentos mais saudáveis. Esses fatores possuem relação entre eles, pois o nível de escolaridade implica, além do conhecimento e da adoção de atividades de autocuidado, a situação ocupacional e as condições de trabalho. Estas, por sua vez, refletem a situação econômica, a qual pode delimitar o acesso a bens como a saúde, o que elucida o ajuste do modelo das variáveis sociodemográficas pela renda familiar e classe econômica (Arruda et al., 2017).

O consumo de bebida alcoólica estava presente em 37,28\% (tabela 3) dos que responderam ao questionário. A Pesquisa Nacional de Saúde (PNS), feita em 2013 pelo Instituto Brasileiro de Geografia e Estatística (IBGE), demostrou que ao verificar o consumo de álcool por sexo, o uso pelos homens foi de 36,3\%, enquanto pelas mulheres foi de $13,0 \%$, sendo que o intervalo de faixa etária que mais utiliza o álcool é o de 25 a 39 anos.

O consumo de álcool torna o ser humano mais exposto às doenças cardiovasculares, neoplasias, absenteísmo, aposentadorias precoces, acidentes de trabalho e de transporte, episódios de violência, homicídios, suicídios e elevada frequência nos leitos hospitalares (Albuquerque \& Farias, 2019).

O tabagismo como doença neurocomportamental de contágio social é um dos principais motivos de morbimortalidade que poderia ser evitável no mundo. Estima-se que cerca da metade dos indivíduos que fazem uso do tabaco morrerá de alguma doença relacionada ao mesmo (Monteiro, et.al., 2019). 
Tabela 3. Caracterização da condição de saúde em relação ao fumo e bebidas alcoólicas, Imperatriz - MA, 2021.

\begin{tabular}{lcc}
\hline Variável & N & Percentual (\%) \\
\hline Fumante & 10 & 5,92 \\
Sim & 159 & 94,08 \\
Não & 169 & 100 \\
Total & & \\
Bebida alcoólica & 106 & 62,72 \\
Sim & 63 & 37,28 \\
Não & 169 & 100 \\
Total & & \\
\hline
\end{tabular}

Fonte: Autores.

Sobre sua autopercepção (Tabela 4) em relação a saúde 76,93\% afirmaram ser ótima/boa. Isso demonstra que a construção da masculinidade, tais como a invencibilidade, capacidade de exposição a riscos, status de dominador, ativo e fornecedor da família, colabora para dificultar a adesão de ações promotoras de saúde, fazendo com que muitos homens se declarem saudáveis, mesmo sem reconhecerem seu estado de saúde (Vieira et.al., 2013).

Em relação a concepção da alimentação, 55,62\% responderam ter uma alimentação saudável e 44,38\% declaram ter uma alimentação não saudável.

Tabela 4. Descrição da Autopercepção de saúde e alimentação, Imperatriz - MA, 2021.

\begin{tabular}{lcc}
\hline Variável & N & Percentual (\%) \\
\hline Classificação da saúde & & \\
Ótima/Boa & 130 & 76,93 \\
Regular & 37 & 21,89 \\
Ruim & 2 & 1,18 \\
Total & 169 & 100 \\
Concepção da alimentação & & \\
Alimentação saudável & 94 & 55,62 \\
Não saudável & 75 & 44,38 \\
Total & 169 & 100
\end{tabular}

Fonte: Autores.

De acordo com a pesquisa de Assumpção et.al., 2017, as mulheres possuem uma melhor qualidade da dieta e isto tem sido atribuída a diversos fatores. As mulheres são mais preocupadas com a qualidade dos alimentos e tendem a investigar com maior atenção as informações nutricionais contidas nos rótulos de alimentos. A pressão em ambiente de trabalho, stress e a necessidade de se alimentar rápido propicia a busca por lanchonetes de fast foods, bares, botecos e barracas de rua, que podem colaborar para má alimentação dos homens.

De acordo com Guerra, et.al., 2018, encontrou-se que alguns dos fatores de risco mais importantes para o desenvolvimento de Doenças Cardiovasculares são o tabagismo, o sedentarismo, o sobrepeso, o consumo de bebidas alcoólicas e a má alimentação associada ao consumo excessivo do álcool.

Quando questionados se possuem posto de saúde localizados próximos a sua residência, 78\% responderam que sim, mas encontra partida 40,24\% não buscaram o serviço de atenção primaria, mesmo tendo uma próxima a sua residência (Tabela 5). 
Tabela 5. Relação da localização da Unidade Básica de Saúde e a procura por atividades no serviço, Imperatriz - MA, 2021.

\begin{tabular}{lcc}
\hline Variável & N & Percentual (\%) \\
\hline Residência é próxima da UBS & 132 & 78,11 \\
Sim & 37 & 21,89 \\
Não & 169 & 100 \\
Total & 65 & 38,46 \\
Sim & 104 & 61,54 \\
Não & 169 & 100 \\
Total & & \\
Procura pela UBS nos últimos 12 meses & 86 & 50,88 \\
Até 3 vezes & 15 & 8,8 \\
Mais de 3 vezes & 68 & 40,24 \\
Nenhuma & 169 & 100 \\
Total & & \\
\hline
\end{tabular}

Fonte: Autores.

Conforme Morais et.al., 2020, a unidade básica de saúde possui como característica um sistema complexo de ações de saúde que engloba a promoção, prevenção, proteção e tratamento o que torna fundamental sua proximidade da população. Segundo Carneiro (2019), os homens quando não possuem nenhum problema de saúde aparente ou que incomode, acreditam não haver necessidade de procurar pelos serviços de atenção primária.

Tem-se como causa que existe em todo o país, um problema nos gestores na concepção de mecanismos para a aproximação e participação dos homens nos serviços de saúde. Como estratégia para deliberar esse entrave o Ministério da Saúde (2009) propõe que sejam incorporadas nas práticas de gestão dos serviços de saúde uma nova filosofia referencial ética, teórica e política, principalmente, relacionado às questões de gênero, bem como que seja ampliado o diálogo entre universidades, profissionais e gestores, a fim de promover a valorização da contextualização crítica e histórica das nuances da atenção à saúde do homem (Pereira \& Nery, 2014).

Conforme Lima e Aguiar (2020), em algumas unidades básicas de saúde foram feitas ações voltadas ao público masculino a partir de exames de sangue, aferição de pressão, mas constataram que os homens não apareceram, o que desmotivou a equipe. Em outra unidade, no mês de novembro na campanha do novembro azul, foram efetuadas palestras com temáticas masculinas, mas poucos compareceram.

Prevenção de doenças e a promoção da saúde são vistas como algo inerente às mulheres, podendo ser compreendidas como parte da concepção tradicional de gênero, em que o cuidado e a preocupação com a saúde estão ligados ao gênero feminino (Carneiro, 2019). Uma das alternativas de acordo com Nery (2013), torna-se imprescindível esboço de novas alternativas para o atendimento efetivo das particularidades da população do gênero masculino, pois a falta aceitação dos serviços de saúde não pode ser relacionado apenas a questões de gênero.

Quando interrogados sobre qual serviço buscam, 42,60\% afirmaram procurar hospital e apenas 20,70\% a Unidade Básica de Saúde. Por outro lado, 53,85\% responderam que buscam a UBS somente quando sentem sinal/sintoma, 39,05\% afirmam que procuram para imunização (Tabela 6).

Dentre os motivos que interferem para que os homens busquem os serviços de saúde na Unidade Básica de Saúde, $59,76 \%$ afirmaram não haver motivos que interfira, 22,49\% responderam que a falta de tempo é o empecilho (Tabela 6). 
Tabela 6. Motivo da busca pelo serviço de saúde de acordo com o nível de complexidade, atendimento médico, frequência da procura e motivo da não procura pelo serviço de APS, Imperatriz - MA, 2021

\begin{tabular}{lcc}
\hline Variável & N & Percentual (\%) \\
\hline Procura atendimento quando precisa & & 42,60 \\
Atenção Terciaria (Hospital) & & \\
Atenção Secundaria (UPA) & 60 & 35,50 \\
Unidade Básica de Saúde & 35 & 20,71 \\
Sem informação & 2 & 1,18 \\
Total & 169 & 100 \\
Procura pela UBS & & \\
Sentiu algum sintoma & 91 & 53,85 \\
Acompanhamento de doença & 5 & 2,96 \\
Imunização & 66 & 39,05 \\
Outro & 7 & 4,14 \\
Total & 169 & 100 \\
Qual a frequência em que busca atendimento médico & & \\
Sempre & 19 & 11,24 \\
Dificilmente & 78 & 46,15 \\
Sempre que sente algum sinal ou & 72 & 42,60 \\
sintoma & 169 & 100 \\
Total & & \\
Motivo da não procura do serviço de saúde & & \\
Falta de tempo & 38 & 22,49 \\
Prefere se automedicar & 17 & 10,06 \\
Outros & 13 & 7,69 \\
Nenhum motivo & 101 & 59,76 \\
Total & 169 & 100 \\
\hline
\end{tabular}

Fonte: Autores.

De acordo com Moreira e Carvalho (2016), são que os homens preferem atendimento em serviços de alta complexidade, porque quando apresentam sinais de agravos encontram nesse serviço ação rápida e curativista. Homens entendem a funcionalidade da UBS como espaço de realização de atendimentos de menor complexidade e exames preventivos. Entretanto, esses indivíduos ainda procuram os serviços emergenciais pela (in)disponibilidade de tempo ou por julgarem a assistência de melhor qualidade (Barreto et al., 2015).

Corroborando com o estudo de Vieira et.al., 2013, existem fatores que interferem na autopercepção do homem sobre o seu estado de saúde, como a baixa escolaridade, a opinião dos serviços de saúde que são ofertados. Outro fator muito importante é a falta de informação acerca dos serviços prestados na UBS e o tempo de realização dos mesmos, que induz os usuários a procurar outro serviço de maior rapidez.

As principais queixas consideradas pelos homens estão relacionadas a sintomas agudos que impedem as atividades diárias em seu trabalho, havendo uma apreensão em ausentar de suas atividades laborais, mesmo com o fornecimento de um atestado médico, isto justifica a procura por médico ser sempre que sente algum sinal/sintoma (Lopes et al., 2017).

Apesar da maior vulnerabilidade e da alta taxa de morbimortalidade, os homens não procuram a UBS, em comparação as mulheres, permitindo muitas vezes alguma complicação que seria vista como passíveis de sanar se agravar. E findam por atribuir a falta de tempo para comparecem aos serviços de saúde ao trabalho (Soares, 2019). De acordo com Silva et al, 2020, os motivos encontrados no seu trabalho foram três: medo de descobrir doenças, vergonha de se expor e não acreditarem que os homens adoecem.

Em relação a satisfação do atendimento que teve quando buscou a UBS, 79,29\% afirmaram gostar e 20,71 não gostaram (Tabela 7). 
Tabela 7. Satisfação do atendimento recebido na UBS, Imperatriz - MA, 2021.

\begin{tabular}{lcc}
\hline Variável & N & Percentual (\%) \\
\hline Gostou do atendimento & & \\
Sim & 134 & 79,29 \\
Não & 35 & 20,71 \\
Total & 169 & 100 \\
\hline
\end{tabular}

Fonte: Autores.

Com base na tabela 7, 79,29\% dos homens afirmaram gostar do atendimento na Unidade Básica de Saúde, mas como ainda encontram resistência para procurar tais serviços, é fundamental que os homens reconheçam quando estão doentes e que possam aderir a prevenção ofertadas nas unidades (Sousa \& Aguiar, 2020).

\section{Conclusão}

Portanto, diante de todos os dados obtidos, fica evidente que o público masculino tem dificuldade em buscar os serviços básicos de saúde como método preventivo, ou para resolutividade de problemas de saúde que é de nível primário, mesmo tendo uma boa percepção do atendimento. Nesse contexto, há necessidade de implantação de políticas públicas mais efetivas com o intuito de estimular os homens a procurar a Atenção Primária de Saúde, assim como criar estratégias para captação desse público, como por exemplo, estender o horário de serviço das Unidades Básicas de Saúde, ter um local direcionado para atendimento da população masculina e realização de educação em saúde permanente com o foco na conscientização.

Diante disso, espera-se que esse trabalho provoque repercussão acerca de tal temática para desenvolvimento de pesquisas com esse tema e melhorias em relação ao acesso dos homens aos serviços de saúde.

\section{Referências}

Albuquerque, F. G. F., \& Farias, M. C.A.D. (2019). Características sociodemográficas de homens atendidos na Atenção Primária à Saúde. Temas em Saúde, $19(3), 2447-2131$.

Arruda, G. O.; Mathias, T. A. F.; \& Marcon, S. S. (2017). Prevalência e fatores associados à utilização de serviços públicos de saúde por homens adultos. Ciência \& Saúde Coletiva, 22, 279-290.

Assumpção, D. D., Domene, S. M. Á., Fisberg, R. M., Canesqui, A. M., \& Barros, M. B. D. A. (2017). Diferenças entre homens e mulheres na qualidade da dieta: estudo de base populacional em Campinas, São Paulo. Ciência \& Saúde Coletiva, 22, 347-358.

Barbosa, Y. O., Menezes, L. P. L., \& de Jesus Santos, J. M. (2018). Acesso dos homens aos serviços de atenção primária à saúde.

Carneiro, V. S. M., Adjuto, R. N. P., \& Alves, K. A. P. (2019). Saúde do homem: identificação e análise dos fatores relacionados à procura, ou não, dos serviços de atenção primária. Arquivos de Ciências da Saúde da UNIPAR, 23(1).

Coelho, M. T. Á. D., Rocha, D. M. P., \& da Silva Carneiro, R. A. (2017). Influência da masculinidade nas concepções e práticas de saúde-doença de alunos da educação superior em saúde. Interfaces Científicas-Humanas e Sociais, 6(1), 47-58.

Czorny, R. C. N., Gazetta, C. E., Pinto, M. H., Ribeiro, R. C. H. M., Beretta, D., \& Rodrigues, C. C. (2017). Perfil do usuário homem atendido em uma unidade básica de saúde da família. Rev Enferm UFPE, 11(4), 1624-1631.

Fontes, W. D. D., Barboza, T. M., Leite, M. C., Fonseca, R. L. S., Santos, L. C. F. D., \& Nery, T. C. D. L. (2011). Atenção à saúde do homem: interlocução entre ensino e serviço. Acta paulista de enfermagem, 24, 430-433.

Guerra, D. M., Marques, J. G., Da Silva, B. B., Medeiros, A. G., Pinto, F. C. C., \& Bastos, V. P. D. (2018). Análise ergo espirométrica de indivíduos com fatores de risco para doenças cardiovasculares. RBPFEX-Revista Brasileira de Prescrição e Fisiologia do Exercício, 12(79), 985-993.

Lopes, G. S. S. P., Sardagna, M. C., \& Iervolino, S. A. (2017). Motivos que levam os homens a procurar um serviço de pronto atendimento. Enfermagem Revista, 20(2), 151-165.

Marconi, M. A., Lakatos, E. M. (2017). Metodologia do trabalho científico. Atlas.

Monteiro, L. V., Silva, Í. R., Oliveira Moura, C., \& da Silva, M. M. (2019). Significados atribuídos pelo homem acerca da relação entre o tabagismo e o adoecimento por câncer. Cogitare enferm, 24, e61384. 
Research, Society and Development, v. 11, n. 1, e56011124946, 2022

(CC BY 4.0) | ISSN 2525-3409 | DOI: http://dx.doi.org/10.33448/rsd-v11i1.24946

Morais, A. L. D. J., Feitosa Sousa, P. H. S., Almeida, T. F., Valença, D. C., Basso, B. A. G., Santos, C. D. J., \& Gomes, T. M. C. (2020). A qualidade da atenção primária em saúde: uma revisão integrativa. Journal of Health Connections, 10(3).

Moreira, M. A., \& Carvalho, C. N. (2016). Atenção Integral à Saúde do Homem: Estratégias utilizadas por Enfermeiras (os) nas Unidades de Saúde da Família do interior da Bahia. Saúde \& Transformação Social/Health \& Social Change, 7(3), 121-132.

Oliveira Gomes, J. W., de Freitas Azevedo, N., Soares, G. G., Lacerda, R. B., Garcia, L. A. A., \& Gonçalves, J. R. L. (2019). Jornada de Saúde do Homem: relato de experiência. Revista Família, Ciclos de Vida e Saúde no Contexto Social, 7(1), 104-110.

Pereira, L. P., \& Nery, A. A. (2014). Planejamento, gestão e ações à saúde do homem na estratégia de saúde da família. Escola Anna Nery, 18(4), 635-643.

Silva Barreto, M., de Oliveira Arruda, G., \& Marcon, S. S. (2015). Como os homens adultos utilizam e avaliam os serviços de saúde. Revista Eletrônica de Enfermagem, 17(3).

Silva, A. N., Silva, S. A. D., Silva, A. R. V. D., Araújo, T. M. E. D., Rebouças, C. B. A., \& Nogueira, L. T. (2018). A avaliação da atenção primária a saúde na perspectiva da população masculina. Revista Brasileira de Enfermagem, 71, 236-243.

Silva, A., Barbosa, M. G. A., da Rocha, A. A., Carvalho, T. W. S., Oliveira Lins, S. R., \& Souza, A. P. B. (2020). Saúde do homem: dificuldades encontradas pela população masculina para ter acesso aos serviços da unidade de saúde da família (USF). Brazilian Journal of Health Review, 3(2), 1966-1989.

Sousa Lima, C., \& Aguiar, R. S. (2020). Acesso dos homens aos serviços de atenção primária à saúde: uma revisão integrativa. Research, Society and Development, 9(4).

Teixeira, D. B. S. (2016). Atenção à saúde do homem: análise da sua resistência na procura dos serviços de saúde. Revista Cubana de Enfermería, 32(4).

Vieira, K. L. D., Gomes, V. L. D. O., Borba, M. R., \& Costa, C. F. D. S. (2013). Atendimento da população masculina em unidade básica saúde da família: motivos para a (não) procura. Escola Anna Nery, 17, 120-127. 